\title{
Hospitals and Public Health: Conflicting Or Complementing Each Other
}

\author{
Inayat $\mathrm{H}$. Thaver, Nadia Khalid
}

Hospitals - the original facilities for the sick date back to the temples dedicated to "healing gods" in Egyptians and Greeks. The oldest architectural evidence can be dated back to $9^{\text {th }}$ Century AD in Sri Lanka. The word "hospital" comes from the Latin word "hoses" for host or "hospitium" meaning a place to entertain ${ }^{1}$. While medical schools were established in Greece in the 6th Century BC, there is consensus that the first teaching hospital was founded at Gondisapur in present day Iran in 300 AD Ref). Similarly, the evolution of Public Health can be traced to the origins of various religions wherein purity, cleanliness and hygiene had been part of the religion. The Greek and Indus Valley civilization (35001500 BC) also emphasized the sanitation and drainage system and building of wells ${ }^{2}$. However, the concepts of "treating the sick" and "preventing the healthy from getting sick" could not be combined and had been treated as separate entities.

The transition from agrarian society to industrialized society with increase in population including the change in life styles -all have resulted in emergence of both communicable and non-communicable diseases and thus increased morbidity and mortality (the "double burden") ${ }^{3}$. This then followed the "pill for every ill" and "sick care behavior" by the health practitioners, focusing more and more on the treating the diseases either through stationary clinics or through hospitalization. Thus, early days hospital cultures can also be traced back to religious institutions and philanthropy. Some of the countries took the responsibility of maintaining the health of their citizens as one of the social welfare responsibilities; but few in real terms and many only as political slogans. However, due to lack \& improper services, the private for profit and not for profit institutions emerged to take care of the sick and fill that gap ${ }^{4}$. Medical students as well as doctors have been taught to battle disease and treat chronic illness to the end; some efforts for promoting community based education and integrated teaching had been made, but mostly the focus has been on treating the sick. In addition, we have amazing technologies and treatments that have been proven to save lives, but our costs and health outcomes do not seem to match what we pour into the system.

Inayat H. Thaver,
I Brofessor and HOD Department of CHS
Bahria University Medical and Dental College, Karachi
Nadia Khalid,
Senior Lecturer, Department of CHS
I Emria University Medical and Dental College, Karachi
I Received: 04-03-2019
I I I

Traditionally public health professionals are focusing on preventing diseases and maintaining good health. Therefore, they have scorned hospitals as the antithesis of community health. The hospital care remains notably distanced from public health practice and policy ${ }^{5}$. Hospitals consume more than $50 \%$ of health budget and just over a quarter of population have a contact with hospitals ${ }^{6}$. This can be illustrated with the pyramid that is followed in Pakistan including many other developing countries; the community health forms the basis and tip of pyramid as the tertiary care hospitals. However, the allocation of budget is lop-sided, i.e. more money going towards the curative care as compared to basic primary health care ${ }^{7}$. The problem gets compounded and grave when many of the secondary and tertiary care facilities provide the basic primary care mainly due to lack of an effective and operational referral system. Though we do have an effective primary health care program in all the provinces with the Lady Health Workers and Community midwifes and Basic Health Units (BHUs), but the government health facilities with grass root workers are not fully covering many rural, and most of the urban areas, especially the urban squatter areas and peri-urban areas ${ }^{7}$ The urban areas mostly have hospitals which are overcrowded with patients which can easily be managed at primary care settings. The worst scenario is the fact that there is a booming and unregulated private sector including the quacks; the preference for seeking the primary and basic curative care is higher for these practitioners.

Apparently, it appears that doctors working in tertiary care hospitals and public health practitioners focusing on preventive health have conflicting roles. Both the principles and practices of curative and preventive health can play a mutually supportive role if widen our focus of approach to the problem. Unfortunately, in Pakistan the Public Health field has not been fully nurtured (my letter Rise and fall of public health in Pakistan. The role of epidemiologist in infection control at a hospital level is increasing ${ }^{8}$; similarly, identification of an epidemic in the catchment community followed by its management can much better be addressed if we work together. In addition, roles of hospital in PHC has been recognized more than three decades back ${ }^{8}$ and now for achieving Universal Health Care (UHC) in a country, hospital can play a major role. Health education and preventive care sessions can play a crucial role for improving the disease pattern. Above all clinician (who are apparently busy and firefighting) can join hands in conducting research, which can improve not only practices but also overall quality of care of the patients.

The first step must be taken by the clinician to open their doors to public health practitioners, by appreciating their 
roles and including them as part of their team. Reciprocally, the Public Health practitioners should also be seeking the guidance of their fellow clinicians while targeting diseases interventions. It is thus mandatory that this synergy be considered at both policy as well planning and implementation levels. It has been long recognized that prevention of cancer by having screening programmes, early identification of diseases and raising awareness of the people will not only improve the health status but also save the government lot of money ${ }^{9}$ which it would have spent if the preventive services are ignored or side-lined. In addition, all the health cadres, varying from paramedics, nurses, OT technicians, surgeon, specialists etc., can easily apply the public health approach. Let us accept that we get away from a "pill for every ill" approach.

Role of public health practitioners in mass communication, campaigns, raising awareness of the emerging infectious and epidemic diseases cannot be undermined. The preservice training, i.e under-graduate level training of various cadres of health practitioners is mandatory by having more integrated teaching sessions and bringing the relevance and importance of combined approaches.

\section{REFERENCES:}

1. The History of Hospitals and Ward Healthcare Design 2010; 10(3):50-54
2. Samuel Agyei-Mensah, Ama de-Graft Aikins, Epidemiological Transition and the Double Burden of Disease in Accra, Ghana, Journal of Urban Health: Bulletin of the New York Academy of Medicine, 1987; 5:879-897

3. Porter D .Health, civilization and the state: A History of public health from ancient to modern times. New York: Routledge; 1999

4. J Busfield.A pill for every ill': Explaining the expansion in medicine use Social Science \& Medicine. 2010;70(06): 93441

5. J.Wright, F, Antony, A, Philes. Public health in hospitals: the missing link in health improvement. journal of public health medicine 23(3) 153-156.

6. M. McKee and J. Healy. The role of the hospital in a changing environment Bull World Health Organ. 2000; 78(6): 803-810

7. Babar Tasneem Shaikh, Fauziah Rabbani, Najibullah Safi, Zia Dawar. Contracting of primary health care services in Pakistan: Is up-scaling a pragmatic thinking?. J Pak Med Assoc. 2010; 60(5): 387-9.

8. Government of Pakistan. An overview of the Health sector: The way forward. Ministry of Health, Multi Donor Support Unit: Islamabad: 2001

9. Rise and stalling of Public health in Pakistan. Letter to the editor Article in Journal of the Pakistan Medical Association. 2017.

10. Morgan DJ, et al. Role of hospital epidemiologist growing, more resources needed; Infect Control Hosp Epidemiol. 2015 